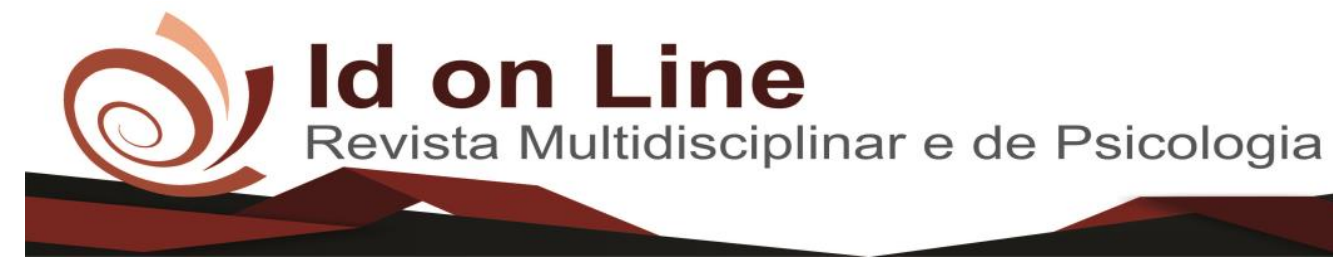

DOI: 10.14295/idonline.v13i48.2178

Artigo

\title{
Prevalência de Incontinência Urinária em Mulheres Praticantes de Pilates e de Musculação
}

\author{
Líllian Freire de Amorim ${ }^{1}$, Daiane Santos Dias Saraiva ${ }^{2}$, Rosana Porto Cirqueira ${ }^{3}$
}

\begin{abstract}
Resumo: A incontinência urinária é um problema que acomete um grande número de pessoas ao redor do mundo. Neste trabalho, pretendeu-se identificar a prevalência de incontinência Urinária em mulheres praticantes de Pilates e de Musculação. É um estudo de caráter exploratório, descritivo e transversal, que foi realizado em uma academia na cidade de Planalto - Ba com mulheres praticantes de pilates e de musculação. Os dados foram colhidos após a aprovação do Comitê de Ética em Pesquisa (CEP) da Faculdade Independente do Nordeste - FAINOR. Aplicaramse questionários com o objetivo de obter dados socioeconômicos e demográficos da população estudada, além de coletar informações referentes à existência de possíveis incontinências urinárias, seja por esforço ou urgência, em mulheres jovens ativas, através do questionário validado para o português ICIQ-SF. Após a coleta dos dados foi feito tratamento estatístico com o objetivo de obter uma análise descritiva com cálculo de porcentagem e das frequências simples, que seguem apresentados em gráficos e tabelas. Foram encontrados um índice de IU maior nas praticantes de musculação, porém, as praticantes de pilates apresentaram IU de maior impacto. Conclui-se que são necessários mais estudos com amostragens maiores objetivando investigar as relações da IU com este tipo de atividade física. Além disso, mesmo com um número amostral pequeno, este trabalho indica que se tenha mais atenção e cuidado, seja pelos praticantes ou pelos profissionais orientadores de musculação, logo que, a amostra, mesmo com 10 anos a menos em média, quando comparada com a amostra de pilates, se mostrou mais suscetível a IU.
\end{abstract}

Palavras-Chave: Epidemiologia. Incontinência Urinária. Técnicas de exercício e movimento. Treinamento de resistência.

\section{Prevalence of Urinary Incontinence in Women practitioners of Pilates and Bodybuilder}

\begin{abstract}
Urinary incontinence is a problem that affects a large number of people around the world. This study aimed to identify the prevalence of urinary incontinence in women practicing Pilates and Bodybuilding. This is an exploratory, descriptive and cross-sectional study, which was conducted in a gym in the city of Planalto - Ba with pilates and bodybuilding women. Data were collected after approval by the Research Ethics Committee (CEP) of the Independent Faculty of the Northeast - FAINOR. Questionnaires were applied to obtain socioeconomic and demographic data of the studied population, besides collecting information regarding the existence of possible urinary incontinence, either due to effort or urgency, in active young women, through the questionnaire validated for Portuguese ICIQ-SF. After data collection, statistical treatment was performed to obtain a descriptive analysis with calculation of percentage and simple frequencies, which are presented in graphs and tables. A higher UI index was found in bodybuilders, however, pilates practitioners had higher impact UI. It is concluded that further studies with larger samples are needed to investigate the relationship of UI with this type of physical activity. Moreover, even with small sample size, this work indicates that more attention and care should be taken by practitioners or bodybuilding guiding professionals, since the sample, even 10 years younger on average, compared to Pilates sample was more susceptible to UI.
\end{abstract}

Keywords: Epidemiology. Urinary incontinence. Exercise and movement techniques. Resistance training.

\footnotetext{
${ }^{1}$ Graduanda em fisioterapia pela Faculdade Independente do Nordeste (FAINOR). li_freire@ hotmail.com;

${ }^{2}$ Graduanda em fisioterapia pela Faculdade Independente do Nordeste (FAINOR). daydd21@ @otmail.com;

${ }^{3}$ Fisioterapeuta. Docente da FTC e FAINOR. Mestre em Saúde Coletiva - UFBA, Brasil. porto_rosana@ yahoo.com.br.
}

311 Id on Line Rev. Mult. Psic. V.13, N. 48 p. 311-322, Dezembro/2019 - ISSN 1981-1179 


\section{Introdução}

A incontinência urinária (IU) é caracterizada pela queixa da perda involuntária de urina, um problema que acomete milhões de pessoas tendo como alvo principal as mulheres jovens ou idosas, , afetando de forma significativa a qualidade de vida, comgrande constrangimento social, disfunção sexual, além de baixo desempenho profissional (JUSTINA, 2013).

De acordo com a International Continence Society (ICS), a incontinência urinária vem sendo erroneamente interpretada como um processo natural do envelhecimento humano, com isso, é negligenciada por muitos profissionais da saúde. Pode ser classificada em três tipos: incontinência urinária de esforço (IUE), incontinência urinária de urgência (IUU) e incontinência urinária mista (IUM); sendo essas, multifatoriais, envolvendo questões como multiparidade vaginal, cirurgias pélvicas prévias, disfunção dos músculos do assoalho pélvico (MAP), obesidade, tabagismo, exercício físico rigoroso e de alto impacto, dentre outros. (CARVALHO et al; 2014).

Hoje em dia, há muitas opções para o tratamento dessa condição, dentre elas pode-se citar a intervenção cirúrgica e o tratamento medicamentoso, além do tratamento conservador, como a fisioterapia, sendo este, o de primeira escolha e que vem se mostrando muito eficaz (OLIVEIRA et al; 2017).

Além disso, outros métodos, como o pilates, podem ser uma alternativa para o tratamento das disfunções do assoalho pélvico, uma vez que trabalha a conscientização corporal, o que desperta a consciência do assoalho pélvico, além de trabalhar na estabilização de tronco. O pilates trabalha com a respiração a fim de promover estabilização pélvica, mobilidade e alinhamento corporal (SCHRADER et al; 2017). Diferentemente da musculação, que embora traga benefícios para a saúde das pessoas, se praticada de forma excessiva e desorientada, pode se tornar um fator de risco para a IU (SILVA et al; 2018).

Neste trabalho, buscou-se quantificar o índice de Incontinência Urinária em dois grupos de mulheres, um de praticantes de musculação e outro de praticantes do pilates visando identificar em que modalidade há maior prevalência de IU, objetivando compreender o assunto e contribuir para melhor prevenção, identificação e tratamento da IU. 


\section{Materiais e Métodos}

Este trabalho é um estudo descritivo e exploratório, com delineamento transversal e abordagem quantitativa, desenvolvido no município de Planalto, Bahia. A amostra foi representada por 60 mulheres com idade entre 20 e 50 anos praticantes de musculação ou pilates e que não estivessem grávidas, nem tenham passado por nenhum procedimento cirúrgico de órgãos pélvicos.

Foram aplicados dois questionários: o primeiro tem por objetivo coletar dados socioeconômicos e sociodemográficos, como idade, nível de escolaridade, número de partos, tipo de parto e estado civil da população pesquisada; o segundo diz respeito às informações das características das incontinências urinárias, o questionário International Consultation on Incontinence Questionnaire- Short Form (ICIQ-SF), validado para o português por Tamanini et al (2004), desenvolvido para avaliar o impacto da IU na qualidade de vida e a qualificação da perda urinária dos pacientes analisados.

O ICIQ-SF é um conjunto de quatro questões que avaliam a frequência, a gravidade e o impacto da IU, além de um bloco de oito itens relacionados a causas ou situações de IU vivenciadas pelos respondentes. O escore é o resultado da soma das questões 3 , 4 e 5 , variando de 0 a 21 pontos, onde " 0 " demonstra nenhum impacto, "1 a 3 " pontos equivale a um impacto leve, "4 a 6" pontos, impacto moderado, "7 a 9" pontos impacto grave e "10" ou mais pontos impacto muito grave. Quanto maior o escore atingido maior a severidade da perda urinária e o impacto na qualidade de vida (TAMANINI et al; 2014)

Os dados só foram colhidos após apreciação e aprovação do Comitê de Ética em Pesquisa - CEP/Fainor conforme parecer 3.505.756. Após a coleta dos dados, foi feita tabulação e estatística descritiva simples a partir do software Microsoft Excel 365. Após isso, os dados foram analisados, como apresentado a seguir, primeiramente o grupo de praticantes de musculação e, em seguida, o grupo de praticantes de pilates.

\section{Resultados}

A idade das trinta praticantes de musculação entrevistadas variou entre 20 e 43 anos, com média em 32 $\pm 7,4$ anos. Dezesseis mulheres $(53,3 \%)$ entrevistadas tiveram filhos, 
apresentando uma média de $2 \pm 1$ filhos, com mínimo de 1 e máximo de 4 . Sobre o tipo de parto, nove $(30 \%)$ descreveram ter dado a luz via parto cesáreo e sete $(23,4 \%)$ via parto normal. Vale ressaltar que oito mulheres $(26,7 \%)$ praticam outro esporte. Dessas, a metade é mãe. Abaixo, segue a tabela 1 que apresenta os dados sociodemográficos das praticantes de musculação:

Tabela 1. Características sociodemográficas da amostra de praticantes de musculação. Planalto - BA, 2019.

\begin{tabular}{|c|c|c|c|}
\hline Características & $\%$ em resposta & $\mathbf{n}$ & $\%$ \\
\hline \multirow[t]{2}{*}{ Sexo } & 100 & & \\
\hline & Feminino & 30 & 100,0 \\
\hline \multirow{4}{*}{ Faixa etária, anos } & 100 & & \\
\hline & $20-30$ & 13 & 43,3 \\
\hline & $30-40$ & 11 & 36,7 \\
\hline & $\geq 40$ & 6 & 20,0 \\
\hline \multirow[t]{5}{*}{ Estado Civil } & 100 & & \\
\hline & Solteira & 19 & 63,3 \\
\hline & Casada & 8 & 26,7 \\
\hline & Divorciada & 2 & 6,7 \\
\hline & Viúva & 1 & 3,3 \\
\hline \multirow[t]{5}{*}{ Escolaridade } & 100 & & \\
\hline & Ensino & 1 & 3,3 \\
\hline & Fundamental & & \\
\hline & Ensino Médio & 16 & 53,4 \\
\hline & Ensino Superior & 13 & 43,3 \\
\hline
\end{tabular}

Fonte: Dados da pesquisa.

Quanto às praticantes de Pilates, a idade das entrevistadas variou entre 28 e 50 anos, com média em $41 \pm 5,5$ anos. Vinte e duas $(73,3 \%)$ mulheres tiveram filhos, tendo no mínimo 1 filho e no máximo 3 , atingindo uma media de $2 \pm 1$ filhos. Quanto ao tipo de parto, doze mulheres (40\%) tiveram parto normal, enquanto dez $(33,3 \%)$ passaram por parto cesariano. Três (10\%) mulheres praticam outro esporte. 
Tabela 2. Características sociodemográficas da amostra de praticantes de Pilates. Planalto BA, 2019.

\begin{tabular}{|c|c|c|c|}
\hline Características & $\%$ em resposta & $\mathbf{n}$ & $\%$ \\
\hline \multirow[t]{2}{*}{ Sexo } & 100 & & \\
\hline & Feminino & 30 & 100,0 \\
\hline \multirow{4}{*}{ Faixa etária, anos } & 100 & & \\
\hline & $20-30$ & 1 & 3,3 \\
\hline & $30-40$ & 12 & 40,0 \\
\hline & $\geq 40$ & 17 & 56,7 \\
\hline \multirow[t]{4}{*}{ Estado Civil } & 100 & & \\
\hline & Solteira & 8 & 26,7 \\
\hline & Casada & 20 & 66,6 \\
\hline & Divorciada & 2 & 6,7 \\
\hline \multirow[t]{5}{*}{ Escolaridade } & 100 & & \\
\hline & Ensino & 2 & 6,7 \\
\hline & Fundamental & & \\
\hline & Ensino Médio & 15 & 50,0 \\
\hline & Ensino Superior & 13 & 43,3 \\
\hline
\end{tabular}

Fonte: Dados da pesquisa

No que diz respeito a incidência da IU das praticantes de musculação, dezesseis mulheres $(53,3 \%)$ apresentam algum tipo de IU. A média do resultado do ICIQ-SF desse grupo é de $6,8 \pm 2,9$, o que categoriza o impacto do grupo como moderado.

Quanto a incidência da IU nas praticantes de pilates, os dados encontram-se no gráfico 1, a seguir:

\section{Índice de IU}

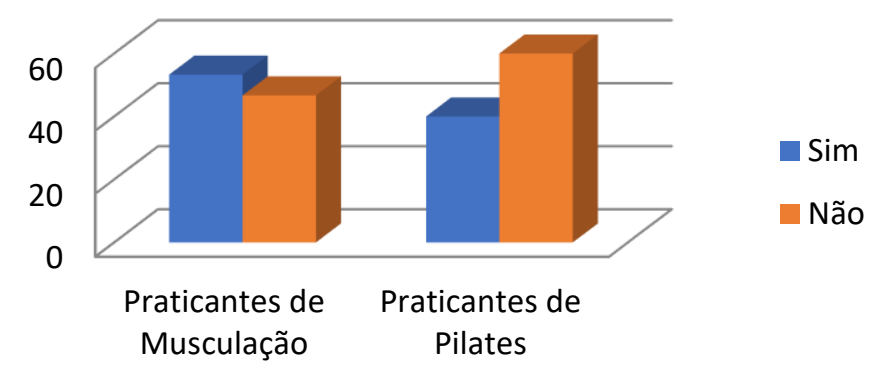

Gráfico 1. Incidência de Incontinência Urinária em praticantes de musculação e em praticantes de Pilates. Planalto - BA, 2019.

Fonte: Dados da pesquisa. 
Doze mulheres (40\%) alega ter alguma IU. A média de resposta desse grupo atingiu 9,5 53,9 pontos no ICIQ-SF, o que sugere um impacto grave.

No que diz respeito ao impacto causado pela IU para as praticantes de musculação, duas informantes $(12,5 \%)$ apresentam impacto leve, seis informantes $(37,5 \%)$ apresentam impacto moderado, cinco $(31,2 \%)$ apresentam impacto grave, e três $(18,8 \%)$ apresentam impacto muito grave.

Já no impacto causado pela IU nas praticantes de Pilates, uma informante $(8,3 \%)$ apresentou impacto leve, duas informantes $(16,7 \%)$ relataram impacto moderado, três mulheres (25\%) disseram sofrer impacto grave e seis entrevistadas (50\%) obteve pontuação que representa impacto muito grave, conforme gráfico 2:

\section{Impacto da IU de acordo ICIQ-SF em praticantes de Musculação e de Pilates}

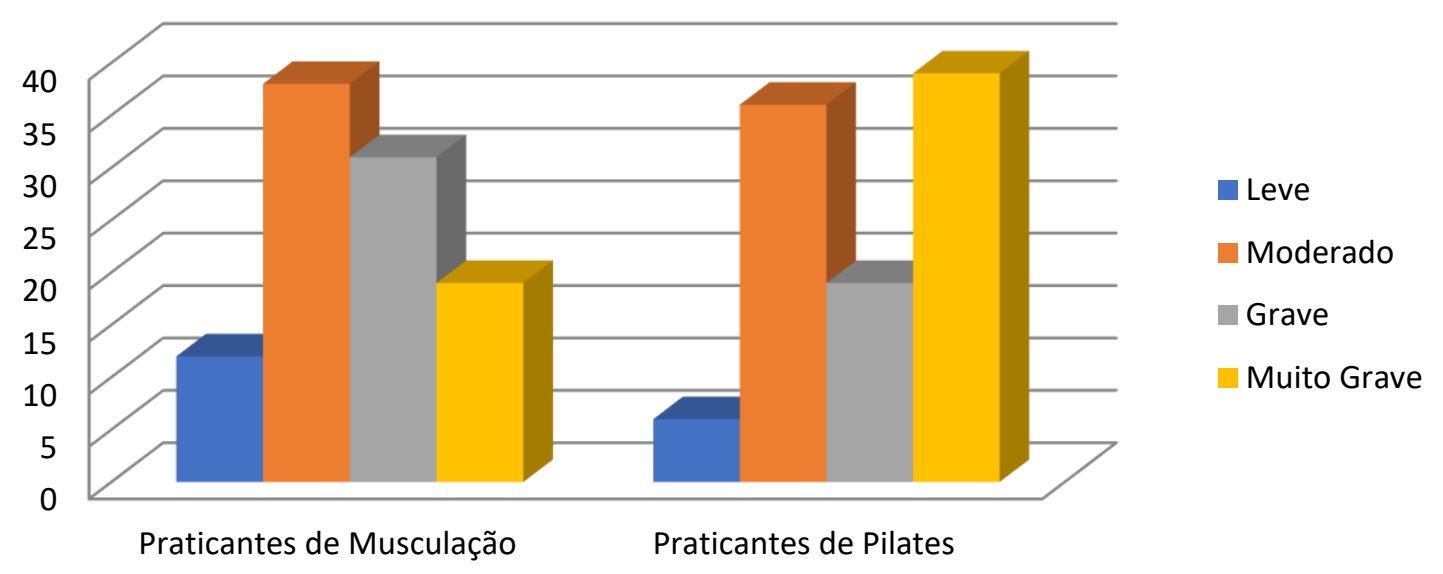

Gráfico 2. Impacto da Incontinência Urinária em praticantes de musculação e praticantes de Pilates. Planalto - BA, 2019.

Fonte: Dados da pesquisa.

Quanto ao tipo de IU, doze (75\%) praticantes de musculação apresentaram incontinência urinária de esforço, três $(18,8 \%)$ incontinência urinária de urgência, e, uma $(6,2 \%)$ incontinência urinária mista. 
Dentre as praticantes de pilates, onze $(91,7 \%)$ relataram ter IU de esforço ao passo que apenas uma informante $(8,3 \%)$ disse ter IU de urgência. Abaixo, segue o gráfico 3 com os dados:

\section{Tipo de IU em praticantes de Musculação e de Pilates, de acordo ICIQ-SF}

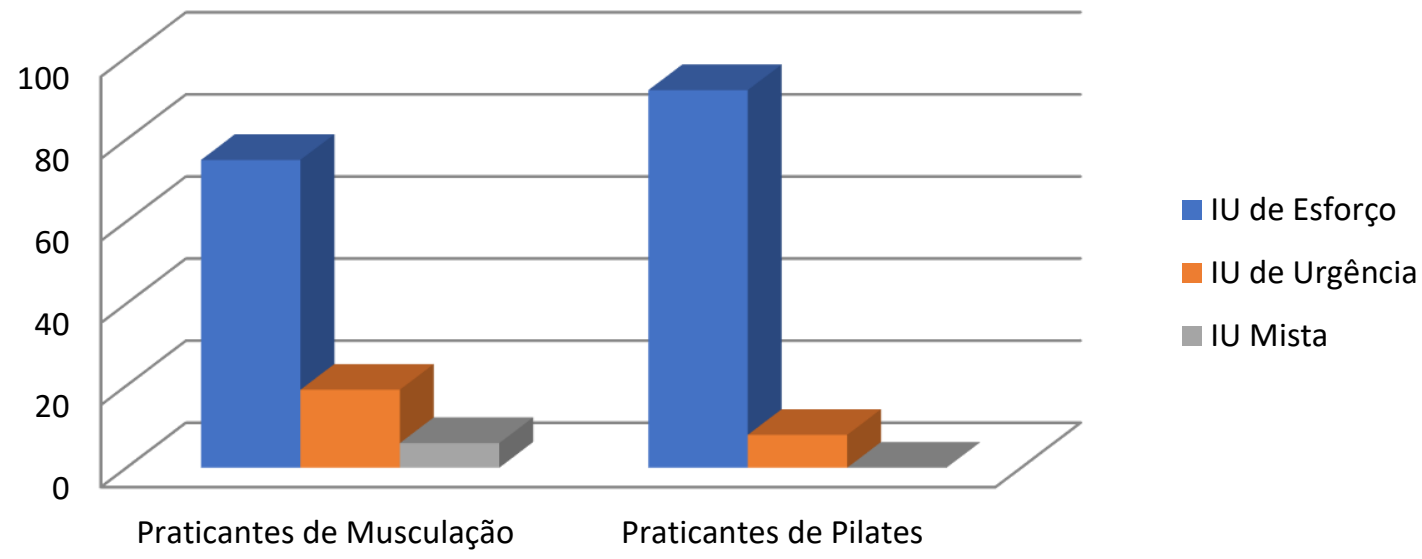

Gráfico 3. Tipo de Incontinência Urinária em praticantes de musculação e de Pilates. Planalto - BA, 2019.

Fonte: Dados da pesquisa.

\section{Discussão}

Neste trabalho foi possível perceber que dentre as praticantes de musculação, mesmo com idade média menor, o índice de IU foi maior do que as praticantes de pilates. Isso vai de encontro, de certa maneira, com alguns teóricos como Latorre e Sperandio (2008). Segundo os autores, o assoalho pélvico, quando enfraquecido, perde capacidade de contração e tonicidade devido a alguns motivos, tais como: parto, aumento da idade, obesidade, deficiência de estrogênio, aumento de pressão intra-abdominal, constipação crônica, entre outros. Nessas situações, as consequências incluem incontinência urinária, redução do prazer sexual e prolapsos (LATORRE; SPERANDIO, 2008). 
Como qualquer ligamento do corpo humano, os ligamentos do assoalho pélvico têm funcionamento equivalente a fortes elásticos, porém, não suportam sobrecargas frequentes. Sendo assim, sempre que há pressão dos órgãos pélvicos em direção céfalo-caudal - como tossir, espirrar, rir, pular - o assoalho pélvico contrai-se fortemente a fim de empurrar os órgãos em direção contrária, evitando o esforço excessivo dos ligamentos (BEREK, 2005).

Estudos como o de Silva et al (2018) concluem que a prevalência de IU em mulheres praticantes de exercícios aeróbicos e musculação é baixa (12\%), com severidade de moderada a grave e sem associação com idade, modalidade, tempo de prática, turno e frequência de exercícios. Poucos estudos foram desenvolvidos com o objetivo em avaliar a relação entre a musculação e a IU. Entretanto, alguns estudos analisam a relação entre IU e atividade física de modo geral, ou principalmente as de alto-impacto.

Diferentemente das conclusões de Silva et al (2018), os resultados deste trabalho apontam para um índice relativamente alto. Mais de 53\% das mulheres praticantes de musculação apresentaram IU, porém, consoante os resultados de Silva et al (2018), 88\% destas mulheres relataram sofrer impacto moderado, grave ou muito grave. Infere-se que, diferentemente do pilates, as mulheres praticantes de musculação possam ter uma menor consciência corporal.

Quarenta por cento das praticantes de pilates relataram ter alguma IU. Vale ressaltar que a idade média das participantes de pilates é 10 anos superior do que a idade média das praticantes de musculação. Esse resultado permite inferir que, mesmo em pessoas de idade mais avançada, o pilates se mostra mais efetivo, corroborando com a ideia de Moreno (2009), Ferreira et al (2015), Pinheiro (2013), dentre outros. No entanto, é possível pensar que, consoante com a literatura, mulheres submetidas a parto vaginal de maneira irregular, apresentam maior prevalência de IU pós-parto quando comparadas a mulheres submetidas à cesárea (LEROY et al.,2016).

O método Pilates tem sido muito utilizado para as disfunções dos músculos do assoalho pélvico (MAP), por ser uma prática que promove baixo impacto, força muscular, flexibilidade, coordenação motora, propriocepção, alinhamento postural, entre outros benefícios. A técnica consiste em exercícios isotônicos e isométricos e tem por característica a grande exigência dos músculos abdominais, músculos estabilizadores da coluna vertebral, íliopsoas, quadríceps, e também da região lombossacral e do assoalho pélvico (MORENO, 2009). 
Durante a prática do Pilates, é preciso que haja uma contração muscular consciente e mantida do centro de força, também conhecido como Core, composto por 29 pares de músculos que suportam o complexo lombo-pelve-quadril. Esses exercícios de contração atinge a pelve fazendo com que o assoalho pélvico também participe dessa contração, associado à respiração, atingindo músculos como o diafragma, músculos do assoalho pélvico, transverso do abdome e os multífidos (FERREIRA et al., 2015).

O assoalho pélvico deve ser forte e flexível para conter os órgãos pélvicos e dar passagem para o feto, para os conteúdos urinários e fecais e também para a vida sexual, porém, as pessoas têm muita dificuldade para ativação da musculatura do assoalho pélvico, o que, muitas vezes, faz com que negligenciem o trabalho dessa musculatura (PINHEIRO, 2013).

No que diz respeito ao impacto causado pela IU nas mulheres, ambos os grupos relataram a ideia de que impacto é grave ou muito grave figurando $50 \%$ das mulheres praticantes de musculação e $58 \%$ das praticantes de pilates.

A incontinência é a disfunção do assoalho pélvico que resulta no desequilíbrio entre as forças de expulsão e as de retenção e ocasiona consequências que variam entre os níveis sociais, psicológicos, econômicos e higiênicos (OLIVEIRA; GARCIA, 2011).

A amostra apontou que a Incontinência Urinária de Esforço foi a maior dentre as possíveis IU. $92 \%$ de praticantes de pilates e $75 \%$ de musculação relataram ter IUE.

A IUE acontece quando, em qualquer aumento da pressão intra-abdominal, ocorre a perda involuntária da urina, como por exemplo: ao tossir, pular, levantar pesos e espirrar; o aumento da pressão intrauretral sobrepõe a pressão intravesical, não sendo assegurada pelos músculos do períneo, resultando na incontinência urinária. Uma das causas mais frequentes é a gravidez, no parto vaginal, no qual a literatura sugere que o feto, pressiona a bexiga, ação que resulta na IUE; e também durante o processo de envelhecimento onde há um enfraquecimento da musculatura do assoalho pélvico (PASCOAL, 2002).

\section{Conclusão}

Chega-se a conclusão, corroborando com o que a literatura apresenta de modo geral, que a prática de Pilates, devido ao enfoque no fortalecimento dos músculos abdominais, o que 
pode ser um elemento de prevenção quanto ao aparecimento de qualquer tipo de IU, enquanto a musculação, talvez por conta de exagero de carga ou de falta de orientação profissional durante a prática, apresentou índices maiores quanto à incidência da IU. Entretanto, ressalta-se que as praticantes do pilates apresentaram pontuação maior do que as praticantes de musculação no ICIQ-SF, ainda que com prevalência menor, o que sugere novos estudos com amostra maior e com associação entre fatores de risco.

Mesmo com um número amostral pequeno, este trabalho indica que se tenha mais atenção e cuidado, seja pelos praticantes ou pelos profissionais orientadores de musculação, logo que, a amostra, mesmo com 10 anos a menos em média, quando comparada com a amostra de pilates, se mostrou mais suscetível a IU.

\section{Referências}

ALVES, A. T. et al. Estudo comparativo da atividade eletromiográfica dos músculos do assoalho pélvico entre mulheres irregularmente ativas e praticantes de musculação. EFDeportes. Revista digital, n 152. Buenos Aires, 2011.

ARAÚJO, M. Poli, et al. Avaliação do assoalho pélvico de atletas: há relação com Incontinência Urinária? Revista Brasileira de Medicina do Esporte, V.21 N.6 p. 442-446, 2015.

BEREK, J. S. Novak: Tratado de Ginecologia Clínica. Rio de Janeiro: Guanabara Koogam, ISBN: 2005.

BERQUÓ, M. et al. Fisioterapia no tratamento da urgência miccional feminina. Revista Femina. V. 41, N.2. P. 107-123, 2013.

CARVALHO, M. P. et al. O impacto da incontinência urinária e seus fatores associados em idosas. Rev. Bras. Geriatr. Gerontol., Rio de Janeiro, 2014.

CORREA, J. N.; MOREIRA, B. P.; GARCEZ, V. F. Ganho de força muscular do diafragma pélvico após utilização dos métodos pilates ou kegel em pacientes com incontinência urinaria de esforço. Revista UNINGÁ Review. Vol.23,n.2,pp.11-17 Jul - Set 2015.

FERREIRA, T. C. R. et al. Avaliação da força muscular do assoalho pélvico em mulheres sedentárias e que praticam atividade física. Revista da Universidade Vale do Rio Verde. V.13, n.2, p. 450 - 464, 2015. 
JUSTINA, L. B. D. Prevalência de incontinência urinária feminina no brasil: uma revisão sistemática. movimento \& saúde. Revista Inspirar. 2013.

LATORRE, G. F. S.; SPERANDIO, F. F. Prevenção das morbidades relacionadas ao enfraquecimento da musculatura do assoalho pélvico feminino: novo horizonte de prevenção Revista Digital - Buenos Aires - Año 12 - № 118 - 2008.

LEROY, Lígia da Silva; LÚCIO, Adélia; LOPES, Maria Helena Baena de Moraes. Risk factors for postpartum urinary incontinence. Revista da Escola de Enfermagem da USP, v. 50, n. 2, p. 200-207, 2016

MELO, A. T. CIRQUEIRA, R. P. Incontinência Urinária em Mulheres praticantes de Musculação. ID on Line. Rev. Mult. Psic. Vol. 12, n.42, Supl. 1, p.525-535. ISSN: 1981-1179 2018 .

MORENO, A. L. Avaliação do assoalho pélvico: fisioterapia em uroginecologia. Segunda Ed. Barueri, SP: Manoli. 2009.

OLIVEIRA, H.G.A. Efeitos do método Pilates nas disfunções do assoalho pélvico: Uma revisão sistemática. 2016. 22f. tcc (Graduação - curso de fisioterapia) UEP, Campina Grande, 2016.

OLIVEIRA, A. H. A. M. et al. Cartilha educativa para mulheres sobre incontinência urinária: concepção e desenvolvimento. Revista Baiana de Saúde Pública v. 41, n. 2, p. 308-323 abr./jun. 2017.

OLIVEIRA, J. R.; GARCIA. R. R. Cinesioterapia no tratamento da incontinência urinária em mulheres idosas. Rev. Bras. Geriatr. Gerontol. Rio de Janeiro, 2014.

PINHEIRO, F. M. Fortalecimento do assoalho pélvico como prevenção da incontinência urinária no período gestacional e pós-parto normal. Ariquemes, 28 de Junho de 2013.

SANTOS, A. M. et al. Método pilates aplicado à aptidão física: uma revisão sistemática. Revista Mackenzie de Educação Física e Esporte. São Paulo, v. 14, n. 1, p. 93-122, jan./jun. 2015 .

SCHRADER, E. P. et al. Eficácia do método Pilates e do biofeedback manométrico em mulheres na menopausa com incontinência urinária. Semina: Ciências Biológicas e da Saúde, Londrina, v. 38, n. 1, p. 61-78, jan./jun. 2017.

SILVA, K.C.C.; FERREIRA, E.G.; ALVES, R.C. Avaliação da prevalência de incontinência urinária em idosas através do questionário de impacto de incontinência urinária (ICIQ-SF). Revista Amazônia Science \& Health. 2014.

SILVA, L. B. et al. Disfunções urinárias em mulheres praticantes de ativididade física em academias - um estudo transversal. Revista Pesquisa em Fisioterapia. V.8 n.1, p.71-78, 2018. 
TAMANINI, J. T. N. et al. Validação para o português do "International Consultation on Incontinence Questionnaire - Short Form" (ICIQ-SF). Revista de Saúde Pública. V. 38, n3. 2004.

\section{Como citar este artigo (Formato ABNT):}

AMORIM, Líllian Freire de; SARAIVA, Daiane Santos Dias; CIRQUEIRA, Rosana Porto. Prevalência de Incontinência Urinária em Mulheres Praticantes de Pilates e de Musculação. Id on Line Rev.Mult. Psic., Dezembro/2019, vol.13, n.48, p. 311-322. ISSN: 1981-1179.

Recebido: 11/11/2019

Aceito: $14 / 11 / 2019$ 